\title{
Ferrimagnetism in the Hubbard, dimmer-connector frustrated chain
}

\author{
J. Rösslel|; D. Mainemer \\ Departamento de Física, Facultad de Ciencias, Universidad de Chile, Casilla 653, Santiago, Chile
}

Received April 21, 2009, in final form February 24, 2010

We study the $\mathbf{A B}_{2}$ "dimmer-connector" chain within a generalized Hubbard model, which contains sitedependent parameters, and different chemical potentials for $\mathbf{A}$ and $\mathbf{B}$ sites. Considering one electron per atom, we carry out exact calculations for finite clusters, and derive some asymptotic results, valid for macroscopic chains. We take a non-vanishing intra-dimmer electron hopping, thus departing from the condition of a bipartite lattice. In spite of that, the system persists ferrimagnetic in some region of the parameter space, thus generalizing a theorem of Lieb for bipartite lattices. A somewhat surprising result is that the ferrimagnetic phase is possible, even for a very large chemical potential jump between $\mathbf{A}$ and $\mathbf{B}$ sites. In another respect, we show that a previously reported macroscopic $\left(2^{N}\right)$ degenerancy of the $\mathbf{A B}_{2}$ Heisenberg chain ground state (GS) is fully removed on going to the (more fundamental) Hubbard model, yielding a non-magnetic GS.

Key words: Hubbard model, Heisenberg model, ferrimagnetic order, frustration, lozenge lattice, dimmer

PACS: 73.90.+f, 75.10.Lp, 75.10-75.25

\section{Introduction}

Although the Hubbard model was introduced in order to explain itinerant ferromagnetism (FM), the appearance of a truly ferromagnetic phase has been somewhat elusive. Nagaoka [1] obtained FM in a very punctual case $(U \rightarrow \infty$ limit and a single hole in a half-filled band). Long range electron hopping may also yield ferromagnetism, even in one dimension [2, 3]; the latter results may be associated with the frustrating effect introduced by the different hoppings.

An important theorem due to E. Lieb [4] establishes the existence of FM in a more ample context, this is the case of a half-filled band system, with the topology of a bipartite lattice $\Lambda=\mathbf{A}+\mathbf{B}$. Under such hypothesis, the net spin of the ground state becomes $S=\frac{1}{2}|| A|-| B||$; here $|A|,|B|$ denote the number of sites in each sublattice. Therefore, a net (macroscopic) magnetic moment exists when the number of sites in the two sublattices differ, $|A| \neq|B|$. The lattice topology is defined by the non-vanishing hopping amplitudes, and in this bipartite case a nonvanishing hopping $t_{a, b} \neq 0$ only occurs when $a \in \mathbf{A}$ and $b \in \mathbf{B}$ or vice versa; otherwise $t_{a, b}=0$.

This "two sublattice ferrimagnetism" of Lieb can be easily understood in the strongly interacting $(U \rightarrow \infty)$ limit, where a half-filled band Hubbard model becomes equivalent to a Heisenberg Hamiltonian with positive exchanges [5]. Accordingly, an antiferromagnetic (AF) coupling exists between the sites of $\mathbf{A}$ and $\mathbf{B}$ sublattices linked by non-vanishing hopping amplitudes, while no coupling exists between the spins lying in the same sublattice. In this way, the spins of the $\mathbf{B}$ sublattice surrounding a given spin of the $\mathbf{A}$ sublattice are antiparallel to such a spin, and therefore, they dispose parallel to each other. Extending this argument to the whole lattice we conclude that each sublattice has an internal ferromagnetic order, while the magnetic moment of the $\mathbf{A}$ and $\mathbf{B}$ sublattices disposes antiparallel one another, thus yielding a ferrimagnetic (instead of ferromagnetic) order. This argument can be applied even to one-dimensional topologies; that is the case of the "lozenge lattice" for example [6 8], where the Hubbard GS is ferrimagnetic in the half-filled band case.

\footnotetext{
*E-mail: jrossler.fisica@gmail.com
} 
We illustrate former concepts by means of the "connector-dimmer" $\mathbf{A B}_{2}$ chain (see figure 1); there the $\mathbf{B}$ sublattice corresponds to the dimmers, while the A sublattice contains the "connectors" of reference [9]. This chain transforms into the bipartite "lozenge" lattice when the hopping amplitude between the $\mathbf{B}$ sites on the same dimmer vanishes (vertical lines in figure 1). In this case, Lieb's theorem [4] ensures that the net spin of the chain is $S=\frac{1}{2} N$, where $N$ is the number of lattice cells.

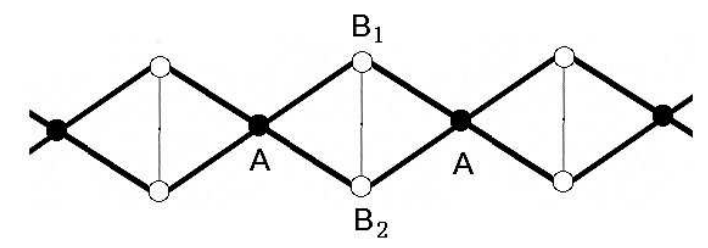

Figure 1. The $\mathbf{A B}_{2}$ "dimmer-connector" chain. The full circles are the connectors, while vertical "dumbbells" are the $\mathbf{B}_{2}$ dimmers.

The $\mathbf{A B}_{2}$ chain has been studied in the context of Heisenberg [9 11] and half-filled band Hubbard [6 6 -8] models. While [9 11] include two antiferromagnetic exchanges, $J$ (connecting A-B sites) and $J_{0}$ (connecting both $\mathbf{B}$ sites on the same dimmer), [6 6 ] only consider a non-vanishing electronic hopping between A and B sites (say, a "lozenge" Hubbard lattice), in order to satisfy Lieb's hypothesis of a bipartite lattice [4]. Lieb's results were generalized, showing that the ferrimagnetic order persists in the "lozenge" and in other one-dimensional lattices, when site dependent Hubbard parameters are considered [7].

On taking the Heisenberg $(U \rightarrow \infty)$ limit, the lozenge Hubbard chain transforms into an alternate arrangement of $s=\frac{1}{2}$ (connector sites) and $s=1$ (dimmer sites) spins with AF coupling $J>0$ (while the intra-dimmer coupling vanishes, $J_{0}=0$ ). In spite of the effect of quantum fluctuations (which are especially relevant in one-dimension), this one-dimensional lattice yields a long range ferrimagnetic order [12], with a total spin per cell $S=\frac{1}{2}$.

\section{The model}

The aim of this contribution is to establish whether the Lieb's FM ground state (GS) persists, if we depart from the bipartite lattice hypothesis by introducing a non-vanishing hopping $t_{0}$ between the two $\mathbf{B}$ sites on the same dimmer (see figure 1). Several actual systems may be described by such kind of lattices [13].

We shall describe the $\mathbf{A B}_{2}$ chain using a generalized Hubbard model, with the associated Hamiltonian

$$
\begin{aligned}
H_{U}= & -\sum_{\ell, \sigma}\left[t\left(a_{\ell, \sigma}^{\dagger}+a_{\ell+1, \sigma}^{\dagger}\right)\left(b_{\ell, 1, \sigma}+b_{\ell, 2, \sigma}\right)+t_{0} b_{\ell, 1, \sigma}^{\dagger} b_{\ell, 2, \sigma}+\text { Hermit. Conj. }\right] \\
& +\sum_{\ell, \sigma}\left[E_{A} a_{\ell, \sigma}^{\dagger} a_{\ell, \sigma}+\frac{U_{A}}{2} a_{\ell, \sigma}^{\dagger} a_{\ell, \sigma} a_{\ell,-\sigma}^{\dagger} a_{\ell,-\sigma}\right] \\
& +\frac{1}{2} U_{B} \sum_{\ell, \sigma}\left[b_{\ell, 1, \sigma}^{\dagger} b_{\ell, 1, \sigma} b_{\ell, 1,-\sigma}^{\dagger} b_{\ell, 1,-\sigma}+b_{\ell, 2, \sigma}^{\dagger} b_{\ell, 2, \sigma} b_{\ell, 2,-\sigma}^{\dagger} b_{\ell, 2,-\sigma}\right]
\end{aligned}
$$

here $a_{\ell, \sigma}^{\dagger}$ and $b_{\ell, j, \sigma}^{\dagger}$ create a spin $\sigma$ electron on sites $\mathbf{A}$ and $\mathbf{B}_{j}(j=1,2)$ respectively, in the $\ell$-th cell (see figure 1). $U_{A}$ and $U_{B}$ are the corresponding Coulomb repulsions, while $E_{A}$ measures the chemical potential on a site $\mathbf{A}$, taking $E_{B}=0$ as reference. Equation (11) is a generalization of the Hamiltonian considered in reference [7], since we introduce an intra-dimmer hopping $t_{0}$ and different chemical potentials for nonequivalent sites, following a quite natural physical requisite. 
This Hamiltonian has several "global" symmetries, such as the (obvious) translational, inversion and spin rotation invariances (see also [7]). Furthermore, $H_{U}$ has a "local inversion symmetry" associated with each dimmer $\ell$. Such a symmetry implies the conservation of the total spin $\vec{S}_{u, \ell}^{2}=S_{u, \ell}\left(S_{u, \ell}+1\right)$ of the "antibonding" state $u_{\ell, \sigma}^{\dagger}=\left(b_{\ell, 1, \sigma}^{\dagger}-b_{\ell, 2, \sigma}^{\dagger}\right) / \sqrt{2}$ on dimmer $\ell$. The components of $\vec{S}_{u, \ell}$ are

$$
S_{x}+i S_{y}=u_{\ell, \uparrow}^{\dagger} u_{\ell, \downarrow} \quad \text { and } \quad S_{z}=\frac{1}{2}\left(u_{\ell, \uparrow}^{\dagger} u_{\ell, \uparrow}-u_{\ell, \downarrow}^{\dagger} u_{\ell, \downarrow}\right) .
$$

This conservation law implies:

(i) For some sites $\ell$, it holds that $S_{u, \ell}=1 / 2$ and $\sum_{\sigma} u_{\ell, \sigma}^{\dagger} u_{\ell, \sigma}=1$. Therefore, those sites always contain an unpaired antibonding electron $u_{\ell, \sigma}^{\dagger}$, although its spin $\sigma$ may fluctuate.

(ii) Otherwise $S_{u, \ell}=0$, and then $\sum_{\sigma} u_{\ell, \sigma}^{\dagger} u_{\ell, \sigma}=\{0,2\}$. In those sites the antibonding states are created or annihilated in pairs, $u_{\ell, \uparrow}^{\dagger} u_{\ell, \downarrow}^{\dagger}$.

These "local" constants of motion (existing for each $\ell$ dimmer), simplify (to some extent) the many-body problem associated with Hamiltonian (1).

On applying an electron-hole transformation, the Hamiltonian $H_{U}$ preserves its general form (excepting for an irrelevant additive constant), but the parameters $t_{0}, E_{A}$ and $E_{B}$ modify as follows: $t_{0} \rightarrow-t_{0}$ and $E_{A}-E_{B} \rightarrow E_{B}-E_{A}+U_{B}-U_{A}$ (remember that we chose $E_{B}=0$ ). The sign of $t$ is irrelevant, since we can change it by introducing a phase factor -1 on the operators $a_{\ell, \sigma}^{\dagger}$. In contrast, the sign of $t_{0}$ is quite relevant, since the lattice has "electronic kinetic frustration" [14] due to the dimmer-connector triangles.

\section{Strong interaction limit}

We first consider the limit case $\left|t_{0}\right| \ll U_{B}$ and $|t| \ll U_{A}+E_{A}, U_{B}-E_{A}$. The latter inequality presupposes $0<U_{A}+E_{A}, U_{B}-E_{A}$, thus ensuring that a neutral configuration (each site accommodating one electron) is the system GS in the limit $|t|,\left|t_{0}\right| \rightarrow 0$. Using a Schrieffer-Wolff transformation, and projecting over the "neutral" Hilbert subspace (where double occupied or empty sites are excluded, hence each site has attached a spin one-half operator), the Hubbard Hamiltonian $H_{U}$ transforms into a Heisenberg Hamiltonian $H_{\mathrm{H}}$ [5]. Generalizing such transformation to the present model, we conclude

$$
H_{\mathrm{H}}=J \sum_{\ell, \sigma} \vec{S}_{A, \ell} \cdot\left(\vec{F}_{\ell-1}+\vec{F}_{\ell}\right)+J_{0} \sum_{\ell, \sigma} \vec{S}_{B 1, \ell} \cdot \vec{S}_{B 2, \ell}
$$

where $\vec{F}_{\ell}=\vec{S}_{B 1, \ell}+\vec{S}_{B 2, \ell}$ is the total spin of dimmer $\ell$. Here the individual, one-half spins $\vec{S}_{A, \ell}$ and $\vec{S}_{B j, \ell}$ are attached to $\mathbf{A}$ and $\mathbf{B}_{j}$ sites, respectively $(j=1,2)$. The exchange energies $J_{0}$ (intra-dimmer) and $J$ (connector-dimmer) are

$$
\begin{aligned}
& J_{0}=\frac{4 t_{0}^{2}}{U_{B}} \quad \text { and } J=2 t^{2}\left(g_{A}+g_{B}\right) \\
& \text { with } \quad g_{A}=\frac{1}{U_{A}+E_{A}} \text { and } g_{B}=\frac{1}{U_{B}-E_{A}},
\end{aligned}
$$

respectively. We note that $\vec{F}_{\ell}^{2}=F_{\ell}\left(F_{\ell}+1\right)$ is a constant of motion for each $\ell$.

The spin Hamiltonian $H_{\mathrm{H}}$ was studied by Niggemann et al. [10], and other authors [9 11]. Introducing the ratio

$$
R \equiv \frac{J}{J_{0}}=\frac{U_{B} t^{2}}{2 t_{0}^{2}}\left(g_{A}+g_{B}\right),
$$

we summarize their conclusions for the GS: 
(i) When $R>1.10$ (small $t_{0}$ case), it holds that $F_{\ell}=1 \forall \ell$. Here the system is in the Kolezhuk "Ferrimagnetic Phase" [12] (labeled as $P_{\infty}$ in the literature [9]), where spins 1 (dimmers) and $1 / 2$ (connectors) alternate, and the antiferromagnetic coupling yields a non-zero global spin $S=N / 2$ (in spite of quantum fluctuations).

(ii) For $0.5<R<1.10$, it holds that $F_{2 \ell}=1, F_{2 \ell-1}=0$, and the system is in the phase of a period two (labeled as $P_{2}$ in the literature [9]). However, the antiferromagnetic exchange $J$ couples the (non-zero) dimmer spin $F_{2 \ell}$ to its neighbors, $S_{A, 2 \ell}$ and $S_{A, 2 \ell+1}$, thus yielding a singlet (non-magnetic) state.

(iii) Finally, for $R<0.5$ (large $t_{0}$ case), the GS corresponds to the state with $F_{\ell}=0$ for all $\ell$ (the so-called $P_{1}$ phase [9]). Here the GS has a degenerancy $2^{N}$, where $N$ is the number of cells. This macroscopic degenerancy is due to the fact that the energy does not depend on the orientation of each "connector" spin $\vec{S}_{A, \ell}$.

However, this degenerancy is removed on going to the (more fundamental) Hubbard model. In fact, on considering the limit case $|t| \ll\left|t_{0}\right| \ll U_{A}, U_{B}$ and applying the fourth order perturbation theory (instead of the second order analysis that leads to equation (2)), we conclude that the low lying energy states can be described by an effective Heisenberg Hamiltonian

$$
H_{e f f}=\sum_{\ell} J_{\mathrm{cc}} \vec{S}_{A, \ell} \cdot \vec{S}_{A, \ell+1}
$$

with a residual connector-connector coupling $J_{\mathrm{cc}}>0$ given by

$$
J_{\mathrm{cc}}=4 t^{4}\left[\frac{1}{U_{A}}\left(g_{A}-g_{B}\right)^{2}+g_{A} g_{B}^{2}+g_{A}^{2} g_{B}+2 g_{A}^{3}+2 g_{B}^{3}\right] \text {. }
$$

This way, the GS becomes non-degenerate, showing the existence of antiferromagnetic short range correlations between the connector spins.

\section{Small cluster calculations}

In order to depart from the $U_{A}, U_{B} \rightarrow \infty$ limit, we analyze the Hamiltonian (11) for a 5-atom (butterfly-like) cluster, consisting of a connector and two neighboring dimmers. In addition, we also consider a 9-atom (3 dimmers, 3 connectors) ring in order to study the particular limit $E_{A} \rightarrow-\infty$. Although such small clusters cannot reproduce the thermodynamic $(N \rightarrow \infty)$ limit, at least they may retain the main physical features of the larger ones. On the other hand, it is easy to obtain the symmetry properties and conserved magnitudes of these small clusters. We mainly focus on the half filling case. We restrict ourselves to the zero temperature case (GS properties), since the phase diagram becomes blurred for a finite cluster at non-zero temperature.

In the case of the butterfly-like cluster with 5 electrons, the associated Hilbert space has a dimension 252, but using group theory techniques the Hamiltonian can be broken into $18 \times 18$ or smaller blocks. In fact, on considering the cluster symmetries (including the "local" inversion at each dimmer), the Hamiltonian group is isomorphic to $\mathcal{D}_{2, d} \otimes S U_{2}$ (here we use the nomenclature by Hamermesh [15]); $S U_{2}$ describes the spin rotational symmetry, classified by the total spin quantum number $S=\left\{\frac{1}{2}, \frac{3}{2}, \frac{5}{2}\right\}$.

Depending on the parameters of the system, the GS can be on the irreducible representations (IR) $\left[B_{1}, S=3 / 2\right],[E, S=1 / 2]$ and $\left[A_{1}, S=1 / 2\right]$. The $B_{1}$ states are odd to local inversion in both dimmers, the $A_{1}$ states are even in both dimmers, while the $E$ states are odd with respect to one dimmer and even with respect to the other one. The associated values of "antibonding spin" are $S_{u, \ell}=1 / 2$ at an odd dimmer, and $S_{u, \ell}=0$ at an even dimmer.

We first consider the Heisenberg $U_{A}, U_{B} \rightarrow \infty$ limit for our "butterfly cluster". Here we can identify the IR $\left[B_{1}, S=3 / 2\right]$ GS as the ferrimagnetic $\left(P_{\infty}\right)$ phase, since this IR corresponds to $F_{1}=1=F_{2}, \vec{F}_{1} \cdot \vec{F}_{2}=+1$ and $\left(\vec{F}_{1}+\vec{F}_{2}\right) \cdot \vec{S}_{A}=-3 / 2$, thus showing the typical ferrimagnetic correlations (the dimmer spins are parallel to each other, and antiparallel to the connector spin). 
In the case where the GS belongs to the IR $[E, S=1 / 2]$, it holds that $F_{1}=1, F_{2}=0$ and $\vec{F}_{1} \cdot \vec{S}_{A}=-1$. Accordingly, this IR corresponds to the $P_{2}$ phase previously described.

Finally, if the GS belongs to the IR $\left[A_{1}, S=1 / 2\right]$, both dimmer spins vanish $F_{1}=0=F_{2}$. Therefore we identify the IR $\left[A_{1}, S=1 / 2\right]$ as the $P_{1}$ phase.

Due to the finite size and shape of the cluster, the phase diagram in the Heisenberg $U_{A}, U_{B} \rightarrow \infty$ limit does not correspond to the $N=\infty$ case described below equation (4). Now the GS belongs to the IR $\left[B_{1}, S=3 / 2\right]\left(P_{\infty}\right.$ phase) for $R>2$, while the IRs $[E, S=1 / 2]$ ( $P_{2}$ phase) and $\left[A_{1}, S=1 / 2\right]$ ( $P_{1}$ phase) are stable for $1<R<2$ and $R<1$ respectively.

We carry out calculations of spin-spin correlations for the GS of the Hubbard model (with finite values of $U_{A}, U_{B}$ ) at the different phases displayed by the system, concluding the results qualitatively similar to those of the Heisenberg model. Accordingly, we can use the former association between the IRs of the GS and the $P_{j}$ phases, $j=\{1,2, \infty\}$. In this way, when the GS has $S=3 / 2$, the system is in the ferrimagnetic $P_{\infty}$ phase, while a GS with $S=1 / 2$ corresponds to a non-magnetic phase (remember that the number of electrons (5) is odd, thus excluding the $S=0$ case).

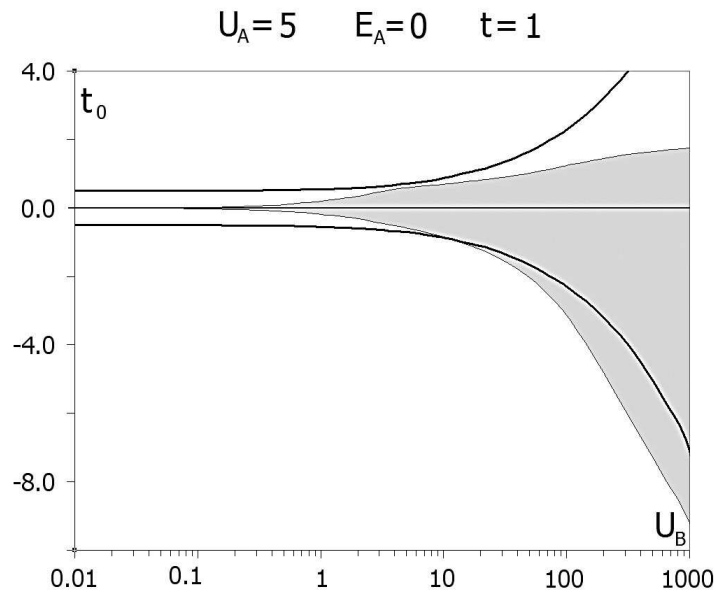

(a)

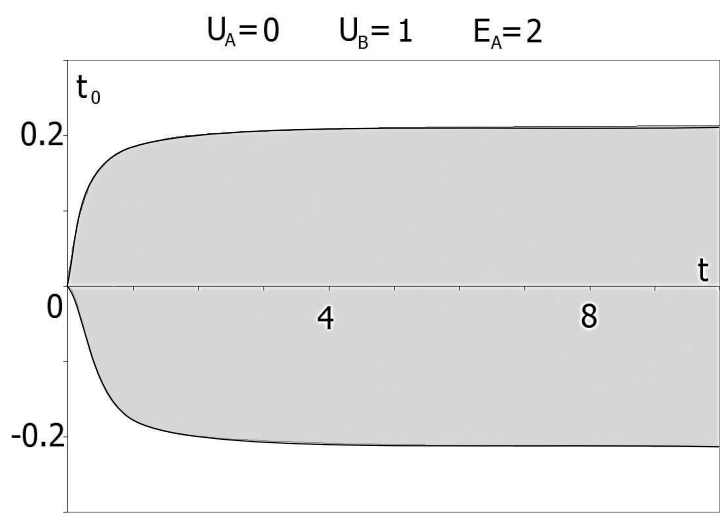

(c)

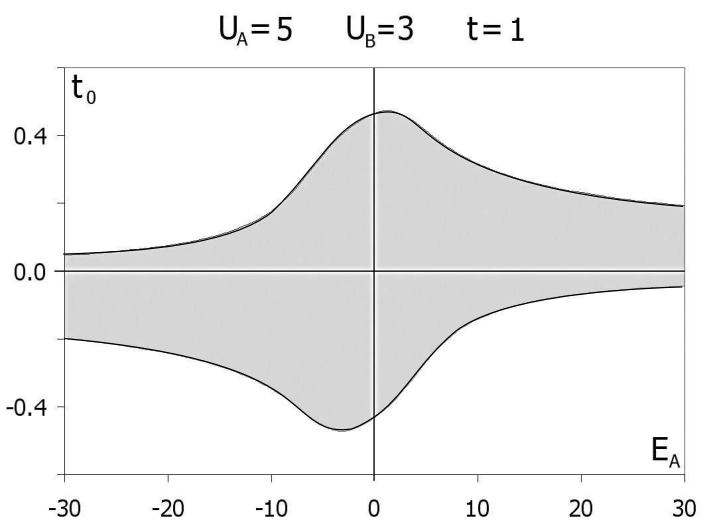

(b)

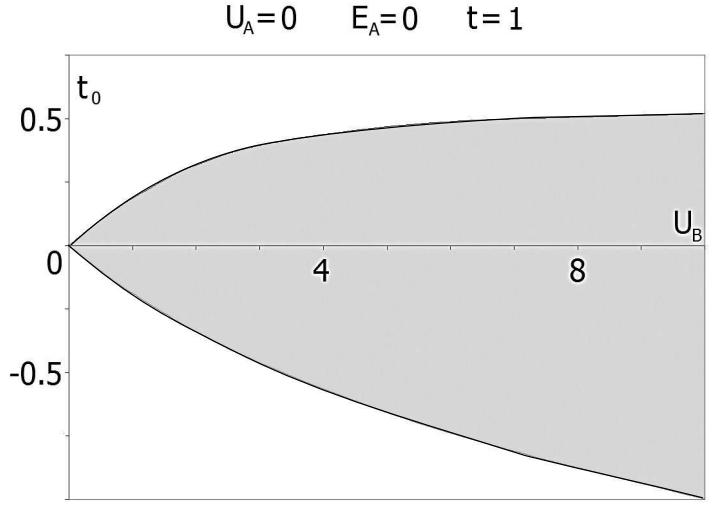

(d)

Figure 2. The phase diagram of the "butterfly cluster" for different parameters of the generalized Hubbard model. The ferrimagnetic region is shown as the shaded area. (a) An $U_{B}$ versus $t_{0}$ plot, with $U_{A}=5, E_{A}=0$, and $t=1$. Here the Heisenberg ferrimagnetic boundary (solid line) is also shown for comparison. A logarithmic scale is used for $U_{B}$. (b) An $E_{A}$ versus $t_{0}$ plot, with $U_{A}=5, U_{B}=3$, and $t=1$. (c) A $t$ versus $t_{0}$ plot, with $U_{A}=0, U_{B}=1$, and $E_{A}=2$. (d) An $U_{B}$ versus $t_{0}$ plot, with $U_{A}=0, E_{A}=0$, and $t=1$. 
The numerical analysis of the "butterfly" Hubbard cluster is summarized in figures 2 (a-d). The shaded area represents the stability region of the ferrimagnetic phase. The main conclusions of our study are:

(a) The description of the system by the Heisenberg Hamiltonian is not suitable, excepting for extremely large values of the Coulomb repulsions $U_{A}, U_{B}$, departing very fast from it as more realistic values are considered. For example, on taking $|t| / U_{A},\left|t_{0}\right| / U_{B} \sim 0.15$, the magnetic-nonmagnetic boundary becomes $J / J_{0} \sim 5$ (instead of the Heisenberg prediction, $J / J_{0}=2$ ). In this way, the "Heisenberg limit" description largely overestimates the size of the magnetic region for small and intermediate values of Coulomb repulsion $U_{B}$, erroneously predicting too large values for $\left|t_{0}\right|$ at the ferrimagnetic-nonmagnetic boundary. However, for larger values of $U_{B}$ and $t_{0}<0$ the Heisenberg description underestimates the size of the ferrimagnetic zone. We compare the Hubbard and Heisenberg magnetic boundaries in figure 2 (a).

(b) While the generalized Lieb's theorem [7] only ensures a ferrimagnetic GS under the hypothesis $t_{0} \rightarrow 0, U_{A}, U_{B}>0$ and $E_{A}=0$, our numerical calculations yield ferrimagnetism well beyond the scope of that theorem, as we shall detail in what follows.

(c) The sign of $t_{0}$ becomes relevant (see figures 2 (a, b)), especially for large values of $\left|U_{A}-U_{B}\right|$. This is due to the "electronic kinetic frustration" [14] and the absence of electron-hole inversion symmetry (only present when $E_{A}=\left(U_{B}-U_{A}\right) / 2$ ).

According to figure 2 (b) the case $t_{0}<0$ favors magnetism for $E_{A}<0$ (say, when the connector corresponds to an anion), while the converse is true for $E_{A}>0$. This is confirmed by the asymptotic relations for the magnetic boundaries:

$$
-\sqrt{U_{B} t^{2} / 2\left|E_{A}\right|}<t_{0}<5 t^{2} / 4\left|E_{A}\right| \quad\left(\text { case } E_{A} \rightarrow-\infty\right)
$$

and

$$
-5 t^{2} / 4 E_{A}<t_{0}<\sqrt{U_{B} t^{2} / 2 E_{A}} \quad\left(\text { case } E_{A} \rightarrow+\infty\right) .
$$

(d) The latter asymptotic relations imply that a ferrimagnetic region is still present in the $\left|E_{A}\right| \rightarrow \infty$ limit, in accordance with figure 2 (b). This is a somewhat surprising behavior, since the charge in the connector is fixed in that limit (the existing zero electrons for $E_{A} \rightarrow+\infty$, and two for $\left.E_{A} \rightarrow-\infty\right)$ and therefore the two dimmers are expected to be disconnected due to the blocking of electron hopping through the connector (as long as charge fluctuations are absent in the connector site).

This behavior seems to be associated with the electron number. In fact, working with 4 (instead of 5) electrons in our "butterfly" cluster, the ferrimagnetic phase disappears for large enough $\left|E_{A}\right|$. We have also considered a 3-dimmer-3-connector ring with 9 electrons (say, one electron per atom) in the $E_{A} \rightarrow-\infty$ limit (which may be important in actual systems with very electronegative bridges connecting magnetic groups). In this case a magnetic region is present for $t_{0}<0$, although it is restricted to a very narrow region of the phase space. At present we are analyzing this point by considering larger clusters and different number of electrons.

(e) A ferrimagnetic GS is also present in the case of zero Hubbard repulsion in the connector site, $U_{A}=0$, as it can be inferred from figures 2 (c, d). Indeed, we have checked that the magnetic-non-magnetic boundary is nearly independent of the $U_{A}$ value.

(f) However, a non-vanishing $U_{B}$ is essential for the appearance of magnetism, since the ferrimagnetic region shrinks to zero as $U_{B} \rightarrow 0$ (see figures 2 (a, d)). The magnetic region increases with $U_{B}$, showing the existence of a linear relation for small values of $U_{B}$. In particular, for a large inter-dimmer kinetic energy $t$, the magnetic region is approximately given by the condition $\left|t_{0}\right|<0.22 U_{B} \ll t$. 
(g) We can summarize the complete phase diagram as follows: There is a narrow ferrimagnetic region (existing for small values of $\left|t_{0}\right|$ ), identified with the $\operatorname{IR}\left[B_{2}, S=3 / 2\right]$, and associated with the $P_{\infty}$ phase of the dimmer-connector Heisenberg chain [9-11]. Here the "antibonding" states are single occupied in each dimmer, $S_{u, 1}=S_{u, 2}=1 / 2$.

For intermediate values of $\left|t_{0}\right|$ the GS lies in the IR $[E, S=1 / 2]$ (associated with the $P_{2}$ phase of the Heisenberg limit). This region is very broad, in comparison to the ferrimagnetic one. Here $S_{u, 1}=1 / 2$ and $S_{u, 2}=0$ in our finite cluster calculations.

Finally, for larger values of $\left|t_{0}\right|$ the IR $\left[A_{1}, S=1 / 2\right]$ (associated with the $P_{1}$ phase [9]) becomes the GS; here $S_{u, 1}=0=S_{u, 2}$. The boundary between the $[E, S=1 / 2]$ and $\left[A_{1}, S=\right.$ $1 / 2$ ] regions is crudely described by the Heisenberg limit condition $R \sim 1$, see equation (44).

\section{Summary}

We have studied the $\mathbf{A B}_{2}$ "dimmer-connector" chain within a generalized Hubbard model, which contains site-dependent parameters. The half filled band case was considered. Our main objective was to determine whether a ferrimagnetic GS persists as we depart from Lieb's theorem hypothesis [4], 7] of a bipartite lattice. For that purpose we introduced a non-vanishing intra-dimmer hopping $t_{0}$. We also included a finite jump in the chemical potential, $E_{A}$, on going from $\mathbf{B}$ to $\mathbf{A}$ sublattice.

We established that the present system has a "local" symmetry at each dimmer, which implies the creation or annihilation of the "antibonding" states in pairs. Therefore the "antibonding" spin, $S_{u, \ell}=\left\{0, \frac{1}{2}\right\}$, is conserved

We first analyzed the small hopping energies, $|t|,\left|t_{0}\right| \rightarrow 0$ and strong Hubbard repulsions limit. In this case the system can be described by a Heisenberg Hamiltonian. We concluded that the GS is ferrimagnetic for $\left(t_{0} / t\right)^{2}<0.45 U_{B}\left(g_{A}+g_{B}\right)$ (see definitions at equation (3)); otherwise the system is non-magnetic. We also showed that a previously reported [9] macroscopic $\left(2^{N}\right)$ degenerancy on the Heisenberg "dimmer-connector" chain is fully removed on going to the Hubbard description.

For finite values of Hubbard repulsions, we carried out small cluster (exact) calculations. We scanned the parameter space, concluding that there always exists a finite interval $t_{0,1}<t_{0}<t_{0,2}$ where the GS is ferrimagnetic. In contrast with the Heisenberg limit, here $\left|t_{0,1}\right| \neq t_{0,2}$. In fact, a negative $t_{0}$ enhances ferrimagnetism if $U_{B} \rightarrow+\infty$ or $E_{A} \rightarrow-\infty$, while a positive $t_{0}$ favors ferrimagnetism in the limit $E_{A} \rightarrow+\infty$ (see figures 2 (a, b)). Indeed, the sign of $t_{0}$ becomes relevant when third order processes in the kinetic energy are considered, and the "electronic kinetic frustration" [14] manifests.

Though a non-vanishing Hubbard repulsion at dimmer sites $\left(U_{B} \neq 0\right)$ is an essential requisite for ferrimagnetism, this phase may persist even in the absence of Coulomb repulsion at connector sites, $U_{A}=0$.

A somewhat surprising conclusion is the persistence of ferrimagnetism, even in the limit $E_{A} \rightarrow$ $\pm \infty$. However, this result is sensitive to the particular electron occupancy.

\section{References}

1. Nagaoka Y., Phys. Rev., 1966, 137, 392.

2. Farkasovsky P., Phys. Rev. B, 2002, 66, 012404;Daul S., Noack R.M., Phys. Rev. B, 1998, 58, 2635.

3. Hiroki Nakano, Yoshinori Takahashi, J. Phys. Soc. Jpn., 2004, 73, 983-990.

4. Lieb E., Phys. Rev. Lett., 1989, 62, 1201.

5. Chao K.A., Spalek J., Oles A.M., Phys. Rev. B, 1978, 18, 3453;Chao K.A., Spalek J., Oles A.M., Phys. Stat. Sol. (b), 1981, 108, 329.

6. Wang W.Z., Bambi Hu, Yao K.L., Phys. Rev. B, 2002, 66, 085101.

7. Tian G.S., Lin T.-H., Phys. Rev. B, 1996, 53, 8196.

8. Macedo A.M.S., dos Santos M.S., Coutinho-Filho M.D., Macedo C.A., Phys. Rev. Lett., 1995, 74, 1851. 
9. Rössler J., Gottlieb D., Phys. Rev. B, 2005, 72, 024443.

10. Niggemann H., Uimin G., Zittarrtz J., J. Phys.: Condens. Matter, 1997, 9, 9031.

11. Takano K., Kubo K., Sakamoto H., J. Phys.: Condens. Matter, 1996, 8, 6405.

12. Kolezhuk A.K., Mikeska H.-J., Shoji Yamamoto, Phys. Rev. B, 1997, 55, R3336.

13. Kikuchi H., Fujii Y., Chiba M., Mitsudo S., Idehara T., Tonegawa T., Okamoto K., Sakai T., Kuwai T., Ohta H., Phys. Rev. Lett., 2005, 94, 227201;Izuoka A., Fukada M., Sugawara T., Mol. Cryst. Liq. Cryst. B, 1993, 232, 103.

14. Haerter J.O., Shastry B.S., Phys. Rev. Lett., 2005, 95, 087202.

15. Hamermesh M., Group Theory. Addison-Wesley Publishing Company, 1964 (see Tables 4-16).

\title{
Феримагнетизм у димер-конекторному фрустрованому ланцюжку Габбарда
}

\author{
Дж. Рьослер, Д. Майнемер \\ Відділ фізики, факультет природничих наук, Університет Чилі, Касілла 653, Сантьяго, Чилі
}

\begin{abstract}
Ми досліджуємо узагальнену модель Габбарда на $\mathbf{A B}_{2}$ "димер-конекторному" ланцюжку, яка містить вузловозалежні параметри і різні хімічні потенціали для вузлів А і В. Розглядаючи випадок одного електрона на вузол, ми виконуємо точні обчислення для скінчених кластерів і отримуємо деякі асимптотичні результати, справедливі для макроскопічних ланцюжків. Ми припускаємо незникаючий інтрадимерний електронний перенос, відходячи таким чином від випадку подвійної гратки (двопідґраткового випадку). Незважаючи на це, система залишається феримагнітною у деякій області простору параметрів, що є узагальненням теореми Ліба для подвійних ґраток. Дещо несподіваним $€$ результат, що феримагнітна фаза можлива навіть для дуже великого стрибка хімічного потенціалу між вузлами А і В. 3 іншого боку, ми показуємо, що раніше вказане макроскопічне $\left(2^{N}\right.$-кратне) виродження основного стану $\mathbf{A B}_{2}$ ланцюжка Гайзенберга цілком знімається при переході до (більш фундаментальної) моделі Габбарда, приводячи до немагнітного основного стану.
\end{abstract}

Ключові слова: модель Габбарда, модель Гайзенберга, феримагнітний порядок, фрустрації, ромбічна гратка, димер 